\title{
Factores de Riesgo de la Disfunción Sexual de las Usuarias del Programa de Planificación Familiar, Hospital Regional Virgen de Fátima, Chachapoyas, 2016
}

\section{Risk factors of the sexual dysfunction of the users of the program of family planning, regional virgin fátima's hospital, chachapoyas, 2016}

\author{
Edwin Gonzáles Paco ${ }^{1}$, Lida Carhuaz Peña ${ }^{2}$, Venancia Jovita De la Cruz Yallico ${ }^{3}$, Nélida Cubas Medina ${ }^{4}$, \\ Gloria Visalot Gonzales ${ }^{5}$, Nancy Smith Carmona Gil ${ }^{6}$, Liliana Guarniz Cusma ${ }^{7}$
}

\section{RESUMEN}

El presente estudio fue de enfoque cuantitativo; de nivel relacional; de tipo: observacional, prospectivo, transversal y analítico, con diseño de casos y controles, cuyo objetivo fue Determinar los factores de riesgo de la disfunción sexual de las usuarias del Programa de Planificación Familiar, Hospital Regional Virgen de Fátima, Chachapoyas, 2016, La muestra estuvo constituida por 60 usuarias, de los cuales 23 presentaron disfunción sexual (casos) y 37 no (controles), Para recolectar los datos se utilizó el "test de funcionamiento sexual femenino", Los resultados evidencian que del 100\% (60) usuarias, el 38,3\% (23) presentaron disfunción sexual, De los factores biológicos: las enfermedades crónicas $(\mathrm{OR}=1,74)$ y el uso de anticonceptivos orales $(\mathrm{OR}=1,91)$ son factores de riesgo de la disfunción sexual, mientras que la Ooferectomía y los anticonceptivos parenterales son considerados factores protectores de la disfunción sexual, De los factores psicológicos: La depresión $(\mathrm{OR}=1,43)$ y la ansiedad $(\mathrm{OR}=1,128)$ son factores de riesgo de la disfunción sexual; mientras que el uso de antidepresivos es un factor protector de la disfunción sexual, De los factores interpersonales: La fatiga $(\mathrm{OR}=1,776)$, abuso sexual $(\mathrm{OR}=$ $1,283)$ y la preocupación $(\mathrm{OR}=6,171)$ son factores de riesgo de la disfunción sexual; mientras que la experiencia sexual y la falta de privacidad son factores protectores de la disfunción sexual.

Palabras claves: disfunción sexual, usuarias, programa, planificación familiar, hospital.

\begin{abstract}
I study the present you went from quantitative focus; Of relational level; Of guy: Observacional, prospective, transverse and analytical, patterned of cases and controls, whose objective was Determining the risk factors of the sexual dysfunction of the users of the Program of Family Planning, Regional Hospital Fátima's Virgin, Chachapoyas, 2016, The sample was composed of 60 users, of the ones that 23 presented sexual dysfunction (cases) and 37 of not (controls), The test of sexual feminine functioning was used in order to gather data, Results evidence than (60) the users, the 38,3\% (23) presented sexual dysfunction, Of the biological factors of the $100 \%$ : Chronic illnesses $(\mathrm{OR}=1,74)$ and the use of oral contraceptives $(\mathrm{OR}=1,91)$ are risk factors of the sexual dysfunction, while the Ooferectomía and the parenteral contraceptives are considered protective factors of the sexual dysfunction, $O f$ the psychological factors: Depression $(O R=1,43)$ and anxiety $(O R=1,128)$ are risk factors of the sexual dysfunction; While the use of antidepressants is a protective factor of the sexual dysfunction, Of the interpersonal factors: Fatigue $(O R=1,776)$, sexual abuse $(O R=1,283)$ and worry $(O R=6,171)$ are risk factors of the sexual dysfunction; While protective factors of the sexual dysfunction are the sexual and short experience of privacy.
\end{abstract}

Keywords: Sexual dysfunction, users, program, family planning, hospital.

\footnotetext{
Dr, Ciencias de Enfermeria, Px-DE, Universidad Nacional Toribio Rodríguez de Mendoza,egp50@hotmail.com

${ }^{2}$ Dra, Lida Carhuaz Peña, Px, TC, Universidad Nacional de Huancavelica, lisam@hotmail.com

${ }^{3}$ Obstetra del Centro de Salud de Chilca - Huancayo, jovitaed@hotmail.com

${ }^{4}$ CCP, Secretaria de la Defensoría Universitaria, Universidad Nacional Toribio Rodríguez de Mendoza cubitas_m@hotmail.com

${ }^{5}$ Lic, Educ, Esp, Lengua Española y Literatura, Secretaria de Escuela Profesional Enfermería, cynthya_150@hotmail.com

${ }^{6}$ Secretaria de la Facultad de Ciencias de la Salud, nancy scg@hotmail.com

${ }^{7}$ Estudiante de la Escuela Profesional de Enfermería, rosy@hotmail.com
} 


\section{INTRODUCCIÓN}

A nivel mundial, El estilo de vida moderno acelerado y competitivo, exceso de roles, alta auto exigencia, situaciones de stress, depresiones, adicciones, son los mayores enemigos de una sexualidad femenina sana y gratificante, Una relación de pareja caracterizada por monotonía, rutina, y más grave aún por descalificaciones, maltrato físico y psicológico, evidencia factores que obviamente van a repercutir en la esfera sexual de manera desfavorable, Es sabido que una pareja que está viviendo una relación muy deteriorada, generalmente suspende su actividad sexual, (Cedrés, 2015, p, 37)

La sexualidad es una parte integral de la personalidad de todo ser humano, Su desarrollo pleno depende de la satisfacción de las necesidades humanas básicas como el deseo de contacto, intimidad, expresión emocional, placer y amor, Los derechos sexuales son derechos humanos universales, La salud sexual debe ser un derecho humano básico para alcanzar una sexualidad saludable, (León \& Cossio, 2010, p, 22).

La disfunción sexual es un trastorno que afecta la sexualidad en su sentido más pleno, tanto de hombres como mujeres, por tanto constituye un problema de salud, que si bien no pone en peligro la vida de las personas, afecta de forma directa la salud física, mental y distorsiona el equilibrio familiar, laboral y social, Sus determinantes pueden encontrarse en la naturaleza humana, desde la constitución biológica, con las consecuencias que sobre la misma producen muchas enfermedades; la naturaleza psicológica, que reclama integración armónica de las potencialidades eróticas dentro de la totalidad del ser; la naturaleza del vínculo de pareja, el ambiente social y cultural ante el erotismo, los aprendizajes, las vivencias de la sexualidad y la afectividad desde los inicios de la vida de las personas, (Munarriz, 2012, p, 36).

La experiencia en sexología clínica adquirida en estos últimos años, indica que es menor el número de mujeres que van a consulta por problemas sexuales propios, que el número de hombres, Esto hace pensar que los problemas se sufren en silencio, o no se consideran problemas, Se conoce que un número importante de mujeres no tienen orgasmo y que son más proclives a perder el interés sexual y apenas lo “conversan entre ellas mismas", (Alfonso, 2010, p, 42).
Universalmente se deduce que la sexualidad, es todo aquello relacionado con las partes, con los sexos, que resultaron de la opción de las especies por el sistema de reproducción sexual como forma óptima de adaptación, supervivencia y evolución, Por tanto, dando un gran salto hasta el momento actual de la humanidad, la sexualidad es todo aquello relacionado con todas las maneras posibles de ser hombre o mujer, en toda su diversidad, La sexualidad no equivale a "conductas sexuales", (Aurole, 2014, p, 56).

Estudios recientes indican que en Inglaterra la disfunción sexual femenina alcanza niveles de prevalencia muy altos, como: $43 \%$ de las mujeres presentan alteraciones en el deseo sexual y un $41 \%$ de cambios psicofisiológicos, (Munarriz, 2012, p, 36),.

La sexualidad de la mujer no está determinada por sus diferencias biológicas con el hombre, sino que involucra factores culturales, sociales, y económicos que tienen su origen en la inequidad de género, Las presiones ejercidas por los riesgos y desigualdades en la vida cotidiana de las mujeres, pueden ocasionar agotamiento físico y psicológico, en particular durante los años de crianza de los hijos, Además, para muchas mujeres el sexo no es siempre un acto voluntario y la primera relación sexual es producto de la coerción de la pareja, a veces en edades tempranas, sin dejar de mencionar las que son víctimas de violación, En consecuencia tienen mayor tendencia que los hombres a desarrollar problemas de ansiedad, depresión y trastornos sexuales (Alayo, 2012, p, 34).

En el Perú, en algunos estudios del Callao, la funcionalidad sexual de las mujeres se traduce en que, el $25 \%$ padecen disfunciones sexuales, el $70 \%$ tienen una función sexual normal y solo el $5 \%$, de trastornos sexuales graves, Asi como del ámbito popular, como del profesional, existe una clara tendencia de asimilar la sexualidad con comportamientos sexuales, sin embargo, la sexualidad descansa en dos pilares esenciales: todo lo relacionado con el dismorfismo sexual original, que el proceso de culturización transforma en el amplio concepto de identidad sexual, de género y todas sus derivaciones; y lo relacionado con la motivación sexual que, el cual se traduce en el deseo erótico (Arias, 2011,p, 45).

Ambos ámbitos están relacionados entre sí, El deseo erótico no deja de ser una dimensión de la identidad sexual, El modo en que construimos y orientamos 
nuestro deseo erótico parte de la manera en que nos sentimos hombres o mujeres, Los comportamientos sexuales funcionales o disfuncionales no son conductas aisladas, si no la expresión del deseo erótico, cuya dinámica se incorpora a la estructura general de personalidad, (Alfonso, 2010, p, 28).

Los comportamientos sexuales compartidos, que articulan el lenguaje erótico, se transforma en señales de comunicación verbal y gestual que se transmiten por los canales propios del sistema establecido entre dos personas, La experiencia erótica humana es de la más psicosomáticas, por tanto, la integridad de sus bases orgánicas psicofisiológicas son también decisivas para comprender las disfunciones sexuales, (Alayo, 2012, p, 64).

En el ámbito local, se realizó un estudio sobre la función sexual femenina en las pacientes asistentes al servicio de planificación familiar del Hospital Regional Virgen de Fátima, en la cual el 53,3\% presentó disfunción sexual quiere decir que se presentó una alteración de la excitación sexual, disminución en la lubricación vaginal, inhibición del orgasmo y satisfacción sexual disminuida, entendiendo que la sexualidad es todo aquello relacionado con todas las maneras posibles de ser hombre o mujer, en toda su diversidad y expresión de su sexualidad, (Gonzales, 2015, p, 63).

Esta alarmante cifra, llama la atención para continuar y elevar el nivel de la investigación hacia los factores de riesgo como determinantes de la disfunción sexual femenina; a la vez haber compartido experiencias en el proceso de recolección de datos con las usuarias del programa de planificación familiar del Hospital Regional Virgen de Fátima.

Por todo lo descrito anteriormente se formuló la siguiente interrogante: ¿Cuáles son los factores de riesgo de la disfunción sexual en las usuarias del Programa de Planificación Familiar, Hospital Regional Virgen de Fátima, Chachapoyas, 2016?.

Las hipotesis fueron: Ha: Los factores de riesgo de la disfunción sexual son los factores biológicos, psicológicos, interpersonales y socioculturales, usuarias del Programa de Planificación Familiar, Hospital Regional Virgen de Fátima, Chachapoyas, 2016, Ho: Los factores de riesgo de la disfunción sexual no son los factores biológicos, psicológicos, interpersonales y socioculturales, usuarias del Programa de Planificación Familiar, Hospital Regional Virgen de Fátima, Chachapoyas, 2016.

Cuyo objetivo general fue Determinar los factores de riesgo de la disfunción sexual en las usuarias del Programa de Planificación Familiar, Hospital Regional Virgen de Fátima, Chachapoyas, 2016.

Este estudio es tan atrayente y a la vez complejo, ya que la misma integra en un todo, lo físico, corporal, emocional y afectivo, Existen factores que inhiben o deterioran una función sexual normal que los convierten en disfunciones sexuales ya sean por aspectos biológicos, entre los cuales se incluye la respuesta genital, el ciclo hormonal y la procreación, con factores psicológicos sean estos anímicos, emocionales, afectivos, a los cuales se suma el tema socio-cultural, que son las ideas, la educación sexual $\mathrm{y}$ actitudes regidas por valores morales que moldean la conducta sexual.

Los resultados del presente estudio sirven para realizar talleres educativos y de intervención a fin de mejorar la calidad de vida y sexual de las mujeres, y de esta manera permitirles gozar y disfrutar de una sexualidad plena, sana y responsable, Además sirve para desarrollar su capacidad de elección libre y responsable de gozar de su sexualidad con igualdad de posibilidades y oportunidades sexuales, Además esta investigación permite seguir elevando el nivel investigativo, donde se desarrolla en condiciones de igualdad de derechos a diferencia de otras mujeres del mundo.

\section{Diseño de investigación}

La presente investigación fue de enfoque cuantitativo; de nivel relacional de asociación de riesgos; de tipo: Según la intervención del investigador fue observacional; según la planificación de la toma de datos fue prospectivo; según el número de ocasiones en que midió la variable de estudio fue transversal y según el número de variables de interés fue analítico, El diseño de estudio fue el epidemiológico Analítico de casos y controles, (Supo, 2014, p, 2).

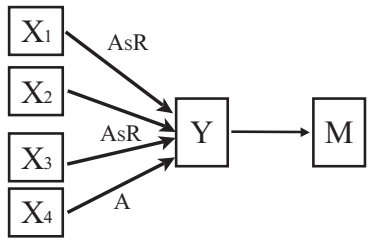


Donde:

M: Muestra de estudio; $\quad \mathrm{X} 1$ : Factores biológicos;

$\mathrm{X} 2$ : Factores psicológicos $\mathrm{X} 3$ : Factores interpersonales

$\mathrm{X} 4$ : Factores socioculturales Y: Disfunción sexual

AsR: Asociación de riesgo

\subsection{Población, muestra y muestreo}

\section{Universo muestral}

El universo muestral estuvo conformado por 60 usuarias de planificación familiar del HRVF; de los cuales 23 presentaron disfunción sexual (casos) y 37 no presentan disfunción sexual (controles).

\section{Criterios de inclusión}

- Todas las usuarias con disfunción sexual (casos) del programa de planificación familiar en el periodo de estudio.

\section{Criterios de exclusión}

- Todas las usuarias sin disfunción sexual (controles) del programa de planificación familiar que no deseen participar del estudio

\subsection{Métodos}

Durante el proceso de investigación, se empleó el método hipotético-deductivo,

\subsection{Técnicas e Instrumentos de Recolección de datos}

Método y técnica: Se utilizó el método de la encuesta y la técnica del cuestionario. (Canales, 1994, p. 224)

Como Instrumento para la $V_{1}$ = Para la variable de los factores; se utilizó una ficha de registro de casos diseñados en una escala dicotómica,

$V_{2}=$ Se utilizó el test de funcionamiento sexual femenino denominado "The Female Sexual Function Index" (FSFI) consistente en 19 ítems, agrupadas en seis dominios: deseo, excitación, lubricación, orgasmo, satisfacción y dolor, El test de funcionamiento sexual femenino fue diseñado y validado por Rosen, R, cuyo valor fue de $\mathrm{VC}=7,998>\mathrm{VT}=1,6449$ y una confiabilidad $\operatorname{Rip}=0,92785$.

\subsection{Procedimiento}

En cuanto al procedimiento de recolección de datos se tuvo en cuenta las siguientes etapas:

1. Se seleccionó y elaboró el instrumento de medición teniendo en cuenta la operacionalización de variables,

2. Se emitió un documento dirigido al Director del Hospital Regional Virgen de Fátima para la respectiva autorización y ejecución de la investigación,

3. Se hizo firmar el consentimiento informado a las mujeres en edad fértil, asistentes al HRVF,

4. Se conversó de forma individual, con cada uno de los participantes explicándole el motivo de la investigación,

5. El instrumento se aplicó a los profesionales de enfermería involucrados en la presente investigación, esta se realizó en forma colectiva,

6. Como última etapa del proceso de recolección de datos se elaboró la tabulación de las mediciones obtenidas para el respectivo análisis estadístico,

\subsection{Análisis de datos}

La información final fue procesada en el software SPSS versión 23, Microsoft Word y Excel, Para contrastar la hipotesis de asociación se utilizó las medidas de asociación de riesgo como la Odds Ratio (OR) y para la hipótesis de relación se sometió a la prueba estadística no paramétrica del ji-cuadrado con un nivel de significancia de $\alpha=$ 0,05 (95\% de nivel de confianza y un 5\% de margen de error). 


\section{RESULTADOS Y DISCUSIÓN}

Tabla 01: Factores de riesgo bilógicos de la disfunción sexual en las usuarias del Programa de Planificación Familiar, Hospital Regional Virgen de Fátima, Chachapoyas, 2016

\begin{tabular}{|c|c|c|c|c|c|c|c|c|c|}
\hline \multirow{3}{*}{\multicolumn{2}{|c|}{$\begin{array}{l}\text { FACTORES } \\
\text { BIOLÓGICOS }\end{array}$}} & \multicolumn{4}{|c|}{ DISFUNCIÓN SEXUAL } & \multirow{2}{*}{\multicolumn{2}{|c|}{ TOTAL }} & \multirow{3}{*}{$\begin{array}{c}\mathbf{X}^{2} \\
\mathbf{p} \text { - valor }\end{array}$} & \multirow{3}{*}{$\begin{array}{l}\text { Odds Ratio } \\
\text { (OR) }\end{array}$} \\
\hline & & \multicolumn{2}{|c|}{$\mathrm{Si}$} & \multicolumn{2}{|c|}{ No } & & & & \\
\hline & & fi & $\%$ & fi & $\%$ & fi & $\%$ & & \\
\hline \multirow{3}{*}{$\begin{array}{l}\text { Enfermedades } \\
\text { crónicas }\end{array}$} & $\mathrm{Si}$ & 4 & 6,6 & 4 & 6,6 & 8 & 13,3 & \multirow{3}{*}{$\begin{array}{c}X^{2}=0,532 \\
P=0,308\end{array}$} & \multirow{3}{*}{1,74} \\
\hline & No & 19 & 31,7 & 33 & 55 & 52 & 86,7 & & \\
\hline & TOTAL & 23 & 38,3 & 37 & 61,7 & 60 & 100 & & \\
\hline \multirow{3}{*}{ Ooforectomía } & $\mathrm{Si}$ & 1 & 1,6 & 2 & 3,3 & 3 & 5 & \multirow{3}{*}{$\begin{array}{l}X^{2}=0,033 \\
P=0,634\end{array}$} & \multirow{3}{*}{0,79} \\
\hline & No & 22 & 36,7 & 35 & 58,3 & 57 & 95 & & \\
\hline & TOTAL & 23 & 38,3 & 37 & 61,7 & 60 & 100 & & \\
\hline \multirow{3}{*}{$\begin{array}{l}\text { Anticonceptivos } \\
\text { orales }\end{array}$} & $\mathrm{Si}$ & 13 & 21,7 & 15 & 25 & 28 & 46,7 & \multirow{3}{*}{$\begin{array}{l}X^{2}=1,455 \\
P=0,320\end{array}$} & \multirow{3}{*}{1,91} \\
\hline & No & 10 & 16,6 & 22 & 36,7 & 32 & 53,3 & & \\
\hline & TOTAL & 23 & 38,3 & 37 & 61,7 & 60 & 100 & & \\
\hline \multirow{3}{*}{$\begin{array}{l}\text { Anticonceptivos } \\
\text { parenterales }\end{array}$} & $\mathrm{Si}$ & 10 & 16,6 & 20 & 33,3 & 30 & 50 & \multirow{3}{*}{$\begin{aligned} \mathbf{X}^{2} & =\mathbf{0}, 635 \\
\mathbf{P} & =\mathbf{0}, 419\end{aligned}$} & \multirow{3}{*}{0,65} \\
\hline & No & 13 & 21,7 & 17 & 28,3 & 30 & 50 & & \\
\hline & TOTAL & 23 & 38,3 & 37 & 61,7 & 60 & 100 & & \\
\hline
\end{tabular}

Fuente: Formulario de factores de riesgo y ficha de disfunción sexual
$\mathbf{H a}=\mathrm{p}<0,05$ (Existe relación)
$\mathbf{H o}=\mathrm{p}>0,05$ (No existe relación)
OR > 1: Factor de riesgo;
$\mathbf{O R}=1$ : No hay asociación;

$\mathbf{O R}<\mathbf{1}$ : Factor protector

En la tabla 01: En función a las enfermedades crónicas del 100\% (60) usuarias de planificación familiar el 86,7\% (52) no presenta enfermedades crónicas, y el 13,3\% (8) sí; el 38,3\% (23) presentó disfunción sexual y el 61,6\% (37) no. De estos El 6,6\% presentó enfermedades crónicas y disfunción sexual; el 31,7\% (19) no presentó las enfermedades crónicas pero sí disfunción sexual; existe un $6,6 \%$ (4) si presentó las enfermedades crónicas pero no disfunción sexual, El valor de la hipotesis de relación fue de $\mathbf{X}^{2}=\mathbf{0 , 5 3 2} ; \mathbf{G I}=\mathbf{1} ; \mathbf{p}=\mathbf{0 , 3 0 8}>\quad=\mathbf{0 , 0 5}$, Y la hipótesis de asociación de $\mathbf{O R}=\mathbf{1 , 7 4}$,

En función a las Ooforectomías: el 95\%(57) no presentó Ooferectomías, y el 5\% (3) sí. El 1,6\% (1) no presentó la Ooferectomía pero sí disfunción sexual; el 36,7\% (22) no presentó Ooforectomia pero sí disfunción sexual; existe un $3,3 \%$ (3) que si presentó la Ooforectomia pero no disfunción sexual, El valor de la hipotesis de relación fue de $\mathbf{X}^{2}=\mathbf{0 , 0 3 3}$; $\mathbf{G I}=\mathbf{1} ; \mathbf{p}=\mathbf{0 , 6 3 4}>\mathbf{= 0 , 0 5}, \mathrm{Y}$ de la hipótesis de asociación fue $\mathbf{O R}=\mathbf{0 , 7 9}$,

En función a los anticonceptivos orales: Del 100\% (60) usuarias de planificación familiar el 53,3\% (32) no utilizaron anticonceptivos orales, pero el $46,7 \%$ (28) si los utiliza.
El $21,7 \%$ (13) que utiliza anticonceptivos orales presenta disfunción sexual; el 16,6\% (10) no utiliza anticonceptivos orales pero si presenta disfunción sexual; el 25\% (15) que usa anticonceptivos orales no presentó disfunción sexual. El valor de la hipotesis de relación fue de $X^{2}=\mathbf{1 , 4 5 5} ; \mathbf{G l}=\mathbf{1} ; \mathbf{p}=\mathbf{0 , 3 2 0}>=$ $\mathbf{0 , 0 5}$. $Y$ de la hipótesis de asociación fue $\mathbf{O R}=\mathbf{1 , 9 1}$.

En función a los anticonceptivos parenterales: El $50,3 \%$ (30) no utiliza anticonceptivos parenterales, pero el otro 50\% (30) si, De estos, el 21,7\% (13) que no utiliza anticonceptivos parenterales sí presenta disfunción sexual; el 16,6\% (10) que sí utiliza anticonceptivos parenterales si presenta disfunción sexual; el 33,3\% (20) que usa anticonceptivos parenterales no presentó disfunción sexual.

El valor de la hipotesis de relación fue de $\mathbf{X}^{\mathbf{2}}=\mathbf{0 , 6 3 5}$; $\mathbf{G l}=1 ; p=0,419>=0,05$. Y .

Para contrastar la hipótesis de asociación la siguiente tabla tetracórica se sometió a la medida de asociación de riesgo cuyo valor fue $\mathbf{O R}=\mathbf{0 , 6 5}$ significa que utilizar anticonceptivos parenterales es un factor protector de la disfunción sexual. 
Tabla 02: Factores de riesgo Psicológicos de la disfunción sexual en las usuarias del Programa de Planificación Familiar, Hospital Regional Virgen de Fátima, Chachapoyas, 2016

\begin{tabular}{|c|c|c|c|c|c|c|c|c|c|}
\hline \multirow{3}{*}{\multicolumn{2}{|c|}{$\begin{array}{c}\text { FACTORES } \\
\text { PSICOLOGICOS }\end{array}$}} & \multicolumn{4}{|c|}{ DISFUNCIÓN SEXUAL } & \multirow{2}{*}{\multicolumn{2}{|c|}{ TOTAL }} & \multirow{3}{*}{$\begin{array}{c}\mathrm{X}^{2} \\
\mathrm{p} \text { - valor }\end{array}$} & \multirow{3}{*}{$\begin{array}{l}\text { Odds } \\
\text { Ratio } \\
\text { (OR) }\end{array}$} \\
\hline & & \multicolumn{2}{|c|}{ Si } & \multicolumn{2}{|c|}{ No } & & & & \\
\hline & & fi & $\%$ & fi & $\%$ & fi & $\%$ & & \\
\hline \multirow{3}{*}{ Depresión } & $\mathrm{Si}$ & 5 & 8,3 & 6 & 10 & 11 & 18,3 & \multirow{3}{*}{$\begin{aligned} X^{2} & =0,289 P \\
& =2,36\end{aligned}$} & \multirow{3}{*}{1,43} \\
\hline & No & 18 & 30 & 31 & 51,7 & 49 & 81,7 & & \\
\hline & TOTAL & 23 & 38,3 & 37 & 61,7 & 60 & 100 & & \\
\hline \multirow{3}{*}{ Ansiedad } & $\mathrm{Si}$ & 10 & 16,7 & 15 & 25 & 25 & 41,7 & \multirow{3}{*}{$\begin{array}{l}X^{2}=0,05 \\
P=1,845\end{array}$} & \multirow{3}{*}{1,128} \\
\hline & No & 13 & 21,6 & 22 & 36,7 & 35 & 58,3 & & \\
\hline & TOTAL & 23 & 38,3 & 37 & 61,7 & 60 & 100 & & \\
\hline \multirow{3}{*}{$\begin{array}{l}\text { Uso de } \\
\text { antidepresivos }\end{array}$} & $\mathrm{Si}$ & 3 & 5 & 5 & 8,3 & 8 & 13,3 & \multirow{3}{*}{$\begin{array}{c}X^{2}=0,003 \\
P=2,425\end{array}$} & \multirow{3}{*}{0,96} \\
\hline & No & 20 & 33,3 & 32 & 53,3 & 52 & 86,7 & & \\
\hline & TOTAL & 23 & 38,3 & 37 & 61,7 & 60 & 100 & & \\
\hline
\end{tabular}

Fuente: Formulario de factores de riesgo y ficha de disfunción sexual

$\mathbf{H a}=\mathrm{p}<0,05$ (Existe relación)

OR $>1$ : Factor de riesgo;
Ho $=\mathrm{p}>0,05$ (No existe relación)

$\mathbf{O R}=\mathbf{1}:$ No hay asociación;
$\mathbf{O R}<\mathbf{1}$ : Factor protector
En la tabla 02 y figura 02: En función a la Depresión que se encuentran dentro de los factores Psicológicos asociados a la Disfunción sexual: Del 100\% (60) usuarias de planificación familiar el 18,3\% (11) si presentó depresión y el $81,7 \%$ (49) no presentó depresión; el 38,3\% (23) presentó disfunción sexual y el 61,7\% (37) no presentaron disfunción sexual.

El 8,3\% presentó depresión y disfunción sexual; el $10 \%$ (6) si presentó depresión pero no disfunción sexual; el 30\% (18) no presentó depresión pero si disfunción sexual; el $51,7 \%$ (31) no presentó depresión y tampoco disfunción sexual.

Para contrastar la hipotesis de relación la siguiente tabla de contingencia se sometio a la prueba estadística no paramétrica del Ji - cuadrado cuyo valor fue de $\mathbf{X}^{2}=\mathbf{0 , 2 8 9} ; \mathbf{G l}=\mathbf{1} ; \mathbf{p}=\mathbf{2 , 3 6}>\mathbf{= 0 , 0 5}$ quiere decir que ambas variables no se relacionan entre sí.

Para contrastar la hipótesis de asociación la siguiente tabla tetracórica se sometio a la medida de asociación de riesgo cuyo valor fue $\mathbf{O R}=\mathbf{1 , 4 3}$ el mismo que significa que las usuarias de planificación familiar que presentan depresión tienen 1,43 veces mayor probabilidad de riesgo de presentar disfunción sexual que aquellas usuarias que no presentan depresión.

En función a la Ansiedad que se encuentran dentro de los factores Psicológicos asociados a la Disfunción sexual: Del 100\% (60) usuarias de planificación familiar el 41,7\% (25) si presentó ansiedad y el $58,3 \%$ (35) no presentó ansiedad; el 38,3\% (23) presentó disfunción sexual y el $61,6 \%$ (37) no presentaron disfunción sexual.

El 16,7\% (10) presentó Ansiedad y disfunción sexual; el 25\% (15) si presentó ansiedad pero no disfunción sexual;

el 21,6\% (13) no presentó ansiedad pero si disfunción sexual; el $36,7 \%$ (22) no presentó ansiedad y tampoco disfunción sexual.

Para contrastar la hipotesis de relación la siguiente tabla de contingencia se sometio a la prueba estadística no paramétrica del Ji - cuadrado cuyo valor fue de $\mathbf{X}^{2}=\mathbf{0 , 0 5} ; \mathbf{G l}=\mathbf{1} ; \mathbf{p}=\mathbf{1 , 8 4 5}>=\mathbf{0 , 0 5}$ quiere decir que ambas variables no se relacionan entre sí.

Para contrastar la hipótesis de asociación la siguiente tabla tetracórica se sometio a la medida de asociación de riesgo cuyo valor fue $\mathbf{O R}=\mathbf{1 , 1 2 8}$ el mismo que significa que las usuarias de planificación familiar que presentan ansiedad 1,128 veces mayor probabilidad de riesgo de presentar disfunción sexual que aquellas usuarias que no presentan ansiedad.

En función al uso de antidepresivos que se encuentran dentro de los factores Psicológicos asociados a la disfunción sexual: Del 100\% (60) usuarias de planificación familiar el 13,3\% (8) si utilizaron antidepresivos y el $86,7 \%$ (52) no utilizaron antidepresivos; el 38,3\% (23) presentó disfunción sexual y el $61,7 \%$ (37) no presentaron disfunción sexual.

El 5\% (3) que utilizaron antidepresivos presentaron 
disfunción sexual; el 8,3 (8) si utilizó antidepresivos pero no disfunción sexual; el 33,3\% (20) no utilizó antidepresivos pero si presentaron disfunción sexual; el 53,3\% (32) no utilizó antidepresivos y tampoco presentó disfunción sexual.

Para contrastar la hipotesis de relación la siguiente tabla de contingencia se sometio a la prueba estadística no paramétrica del Ji - cuadrado cuyo valor fue de $\mathbf{X}^{\mathbf{2}}=\mathbf{0 , 0 0 3} ; \mathbf{G I}=\mathbf{1} ; \mathbf{p}=\mathbf{2 , 4 2 5}>\mathbf{= 0 , 0 5}$ quiere decir que ambas variables no se relacionan entre sí,

Para contrastar la hipótesis de asociación la siguiente tabla tetracórica se sometio a la medida de asociación de riesgo cuyo valor fue $\mathbf{O R}=\mathbf{0 , 9 6} \mathrm{el} \mathrm{mismo} \mathrm{que} \mathrm{significa} \mathrm{que} \mathrm{el} \mathrm{uso} \mathrm{de} \mathrm{antidepresivos} \mathrm{es} \mathrm{un} \mathrm{factor} \mathrm{protector} \mathrm{de} \mathrm{la}$ disfunción sexual.

Tabla 03: Factores de Riesgo Interpersonales de la disfunción sexual en las usuarias del Programa de Planificación Familiar, Hospital Regional Virgen de Fátima, Chachapoyas, 2016.

\begin{tabular}{|c|c|c|c|c|c|c|c|c|c|}
\hline \multirow{3}{*}{\multicolumn{2}{|c|}{$\begin{array}{c}\text { FACTORES } \\
\text { RIESGO INTERPERSONALES }\end{array}$}} & \multicolumn{4}{|c|}{ DISFUNCIÓN SEXUAL } & \multirow{2}{*}{\multicolumn{2}{|c|}{ TOTAL }} & \multirow{3}{*}{$\begin{array}{c}\mathbf{X}^{2} \\
\text { p - valor }\end{array}$} & \multirow{3}{*}{$\begin{array}{l}\text { Odds Ratio } \\
\text { (OR) }\end{array}$} \\
\hline & & \multicolumn{2}{|c|}{$\mathrm{Si}$} & \multicolumn{2}{|c|}{ No } & & & & \\
\hline & & $\mathbf{N}^{\circ}$ & $\%$ & $\mathbf{N}^{\circ}$ & $\%$ & $\mathbf{N}^{\circ}$ & $\%$ & & \\
\hline \multirow{3}{*}{ Fatiga } & $\mathrm{Si}$ & 15 & 25 & 19 & 31.7 & 34 & 56.7 & \multirow{3}{*}{$\begin{array}{l}X^{2}=1.11 \\
P=0.843\end{array}$} & \multirow{3}{*}{1.776} \\
\hline & No & 8 & 13.3 & 18 & 30 & 26 & 43.3 & & \\
\hline & TOTAL & 23 & 38.3 & 37 & 61.7 & 60 & 100 & & \\
\hline \multirow{3}{*}{ Abuso sexual } & $\mathrm{Si}$ & 12 & 20 & 17 & 28.3 & 29 & 48.3 & \multirow{3}{*}{$\begin{array}{l}X^{2}=0.22 \\
P=0.703\end{array}$} & \multirow{3}{*}{1.283} \\
\hline & No & 11 & 18.3 & 20 & 33.4 & 31 & 51.7 & & \\
\hline & TOTAL & 23 & 38.3 & 37 & 61.7 & 60 & 100 & & \\
\hline \multirow{3}{*}{$\begin{array}{l}\text { Experiencia sexual } \\
\text { negativa }\end{array}$} & $\mathrm{Si}$ & 9 & 15 & 25 & 41.7 & 34 & 56.7 & \multirow{3}{*}{$\begin{array}{r}X^{2}=4.671 \\
P=0.0367\end{array}$} & \multirow{3}{*}{0.366} \\
\hline & No & 14 & 23.3 & 12 & 20 & 26 & 43.3 & & \\
\hline & TOTAL & 23 & 38.3 & 37 & 61.7 & 60 & 100 & & \\
\hline \multirow{3}{*}{ Preocupación } & $\mathrm{Si}$ & 16 & 26.6 & 10 & 16.7 & 26 & 43.3 & \multirow{3}{*}{$\begin{array}{l}X^{2}=10.45 \\
P=\mathbf{0 . 0 3 9 9}\end{array}$} & \multirow{3}{*}{6.171} \\
\hline & No & 7 & 11.7 & 27 & 45 & 34 & 56.7 & & \\
\hline & TOTAL & 23 & 38.3 & 37 & 61.7 & 60 & 100 & & \\
\hline \multirow{3}{*}{ Falta de privacidad } & $\mathrm{Si}$ & 7 & 11.7 & 20 & 33.3 & 27 & 45 & \multirow{3}{*}{$\begin{array}{l}X^{2}=\mathbf{3 . 1 9 7} \\
P=\mathbf{0 . 0 6 4}\end{array}$} & \multirow{3}{*}{ 0. 372} \\
\hline & No & 16 & 26.7 & 17 & 28.3 & 33 & 55 & & \\
\hline & TOTAL & 23 & 38.3 & 37 & 61.7 & 60 & 100 & & \\
\hline
\end{tabular}

Fuente: Formulario de factores de riesgo y ficha de disfunción sexual

$\mathbf{H a}=\mathbf{p}<\mathbf{0 , 0 5}$ (Existe relación) $\quad \mathbf{H o}=\mathrm{p}>0,05$ (No existe relación)

$\mathbf{O R}>$ 1: Factor de riesgo; $\quad \mathbf{O R}=1$ : No hay asociación; $\quad \mathbf{O R}<1$ : Factor protector

En la tabla 03: En función a la Fatiga que se encuentran dentro de los factores Psicológicos asociados a la Disfunción sexual: Del 100\% (60) usuarias de planificación familiar el 56,7\% (34) si presenta fatiga, y el 43,3\% (26) no presentó fatiga; el 38,3\% (23) presentó disfunción sexual y el 61,6\% (37) no presentaron disfunción sexual.

E1 25\% (15) si presentó fatiga y disfunción sexual; el 31,7\% (19) si presentó fatiga pero no disfunción sexual; el 13,3\% (8) no presentó fatiga pero sí disfunción sexual; el 30\% (18) no presentó fatiga y tampoco disfunción sexual.

Para contrastar la hipotesis de relación la siguiente tabla de contingencia se sometio a la prueba estadística no paramétrica del Ji - cuadrado cuyo valor fue de $\mathbf{X}^{2}=\mathbf{1 , 1 1} ; \mathbf{G I}=\mathbf{1} ; \mathbf{p}=\mathbf{0 , 8 4 3}>\mathbf{0 . 0 5}$ quiere decir que ambas variables no se relacionan entre sí.

Para contrastar la hipótesis de asociación la siguiente tabla tetracórica se sometio a la medida de asociación de riesgo cuyo valor fue $\mathbf{O R}=\mathbf{1 , 7 7 6}$ el mismo que significa que las usuarias de planificación familiar que presentan las fatigas tienen 1,74 veces mayor probabilidad de riesgo de presentar disfunción sexual que aquellas usuarias que no presentan la fatiga. 
En función al abuso sexual que se encuentran dentro de los factores Psicológicos asociados a la Disfunción sexual: Del 100\% (60) usuarias de planificación familiar el 48,3\% (29) si presentaron abuso sexual, y el 51,7\% (31) no presentó abuso sexual; el 38,3\% (23) presentó disfunción sexual y el $61,7 \%$ (37) no presentaron disfunción sexual.

El 20\% (12) si presentó abuso sexual y disfunción sexual; el 28,3\% (29) si presentó abuso sexual pero no disfunción sexual; el 18,3\% (11) no presentó abuso sexual pero sí disfunción sexual; el 33,4\% (20) no presentó abuso sexual y tampoco disfunción sexual.

Para contrastar la hipotesis de relación la siguiente tabla de contingencia se sometio a la prueba estadística no paramétrica del Ji - cuadrado cuyo valor fue de $\mathrm{X}^{2}=0,22 ; \mathrm{Gl}=1 ; \mathrm{p}=0,703>\alpha=0,05$ quiere decir que ambas variables no se relacionan entre sí.

Para contrastar la hipótesis de asociación la siguiente tabla tetracórica se sometio a la medida de asociación de riesgo cuyo valor fue $\mathrm{OR}=1,283$ el mismo que significa que las usuarias de planificación familiar que fueron víctimas de abuso sexual tienen 1,283 veces mayor probabilidad de riesgo de presentar disfunción sexual que aquellas usuarias que no presentaron abuso sexual.

En función a la Experiencia sexual negativa que se encuentran dentro de los factores Psicológicos asociados a la Disfunción Sexual: Del 100\% (60) usuarias de planificación familiar el 56,7\% (34) tuvieron una experiencia sexual negativa, y el 43,3\% (26) no tuvieron una experiencia sexual negativa; el $38,3 \%$ (23) presentó disfunción sexual y el $61,7 \%$ (37) no presentaron disfunción sexual.

El 15\% (9) si presentó una experiencia sexual negativa y disfunción sexual; el 41,7\% (25) si presentaron experiencias sexuales negativas pero no disfunción sexual; el 23,3\% (14) no presentaron una experiencia sexual negativa pero sí disfunción sexual; el 20\% (12) no tuvieron una experiencia sexual negativa y tampoco disfunción sexual.

Para contrastar la hipotesis de relación la siguiente tabla de contingencia se sometio a la prueba estadística no paramétrica del Ji - cuadrado cuyo valor fue de $\mathrm{X}^{2}=4,671 ; \mathrm{Gl}=1 ; \mathrm{p}=0,0367<\alpha=0,05$ quiere decir que ambas variables si se relacionan entre sí.

Para contrastar la hipótesis de asociación la siguiente tabla tetracórica se sometio a la medida de asociación de riesgo cuyo valor fue $\mathrm{OR}=0,366$ el mismo que significa que las usuarias de planificación familiar que tuvieron una experiencia sexual negativa tienen una probabilidad de 0,366 veces de presentar alguna disfunción sexual que aquellas usuarias que no lo presentaron, Tener una experiencia sexual negativa pareciera que es un factor protector de la disfunción sexual.

En función a la Preocupación que se encuentran dentro de los factores Psicológicos asociados a la Disfunción Sexual: Del 100\% (60) usuarias de planificación familiar el 43,3\% (26) presentaron preocupación, y el 56,7\% (34) no presentaron preocupación; el 38,3\% (23) presentó disfunción sexual y el $61,7 \%$ (37) no presentaron disfunción sexual.

El 26,6\% (16) presentaron preocupación y disfunción sexual; el 16,7\% (10) si presentaron preocupación pero no disfunción sexual; el $11,7 \%$ (7) no presentaron preocupación pero sí disfunción sexual; el 45\% (27) no presentaron preocupación y tampoco disfunción sexual.

Para contrastar la hipotesis de relación la siguiente tabla de contingencia se sometio a la prueba estadística no paramétrica del $\mathrm{Ji}$ - cuadrado cuyo valor fue de $X^{2}=10,45 ; G l=1 ; p=0,0399<\alpha=0,05$ quiere decir que ambas variables si se relacionan entre sí.

Para contrastar la hipótesis de asociación la siguiente tabla tetracórica se sometio a la medida de asociación de riesgo cuyo valor fue $\mathrm{OR}=6,171 \mathrm{el}$ mismo que significa que las usuarias de planificación familiar que tuvieron una preocupación tienen 6,171 veces mayor riesgo de presentar disfunción sexual que aquellas usuarias que no lo presentaron.

En función a la Falta de privacidad que se encuentran dentro de los factores Psicológicos asociados a la Disfunción Sexual: Del 100\% (60) usuarias de planificación familiar el 45\% (27) presentaron preocupación, y el 55\% (33) no presentaron preocupación; el 38,3\% (23) presentó disfunción sexual y el $61,7 \%$ (37) no presentaron disfunción sexual.

El 11,7\% (7) presentaron falta de privacidad y disfunción sexual; el 33,3\% (20) si presentaron falta de privacidad pero no disfunción sexual; el $26,7 \%$ (16) no presentaron falta de privacidad pero sí disfunción sexual; el 28,3\% (17) no presentaron falta de privacidad y tampoco disfunción sexual.

Para contrastar la hipotesis de relación la siguiente tabla de contingencia se sometió a la prueba estadística no paramétrica del Ji - cuadrado cuyo valor fue de $X^{2}=3,197 ; \mathrm{Gl}=1 ; \mathrm{p}=0,064>\alpha=0,05$ quiere decir que ambas variables no se relacionan entre sí.

Para contrastar la hipótesis de asociación la siguiente 
tabla tetracórica se sometió a la medida de asociación de riesgo cuyo valor fue $\mathrm{OR}=0,372 \mathrm{el}$ mismo que significa que las usuarias de planificación familiar que tuvieron falta de privacidad tienen una probabilidad de solo 0,372 veces de presentar disfunción sexual, Finalmente indicar que la falta de privacidad es un factor protector de la disfunción sexual.

\section{DISCUSIÓN}

En la tabla 01 , del $100 \%$ de las usuarias de Planificación Familiar del Hospital Regional Virgen de Fátima, el 38,3\% presentó disfunción sexual, quiere las usuarias de planificación familiar presentan alteraciones en la excitación sexual, disminución en la lubricación vaginal, inhibición del orgasmo y satisfacción sexual disminuida, entendiendo que la sexualidad es todo aquello relacionado con todas las maneras posibles de ser hombre o mujer, en toda su diversidad y expresión de su sexualidad,

Comparando con los estudios de Cáceres, (2012), donde demostró que existe un $30 \%$ de la población con disfunciones sexuales, y el $70 \%$ tiene un funcionamiento sexual normal, Entre los factores de riesgo de la disfunción sexual fueron: el uso de anticonceptivos orales y parenterales con una $\mathrm{OR}=$ 1,86; Las enfermedades crónicas con una $\mathrm{OR}=2,26$, Así mismo comparando con Cajahuanca, (2005), En su estudio Factores de riesgo de la disfunción sexual, en las asistentes al servicio de ginecología del Hospital Departamental de Ica, Sus resultados demostraron que los factores de riesgo de la disfunción sexual son: el uso excesivo de anticonceptivos orales $\mathrm{OR}=1,32$ y finalmente con Portilla, (2008), En su estudio Incidencia y Factores de riesgo de la disfunción sexual en mujeres trabajadoras sexuales del Condominio la Coruña, 2007, Sus resultados indican que: existe una tasa de incidencia de $104 \times 1000$ trabajadoras sexuales, Siendo los factores de riesgo: el uso de los anticonceptivos orales $\mathrm{OR}=1,098$,

Comparando los resultados del presente estudio con los resultados de Cáceres, Portilla y Cajahuanca son similares ya que en dichos estudios los factores de riesgo de la disfunción sexual con mayor predominancia son el uso de los anticonceptivos orales, Mientras que el uso de los anticonceptivos parenterales es similar al estudio de Cáceres, Así mismo las enfermedades crónicas son consideradas como un factor de riesgo.

Así mismo comparando con los estudios de Gonzales, (2015), Perú, En su estudio Determinar la función sexual de las usuarias del Programa de Planificación Familiar, Hospital Regional Virgen de Fátima, Chachapoyas, 2015, Sus resultados evidenciaron que el $53.3 \%$ de las usuarias presentaron disfunción sexual como: alteraciones de la excitación sexual, disminución en la lubricación vaginal, inhibición del orgasmo y satisfacción sexual disminuida. Al comparar con los resultados del presente estudio los resultados son similares, ya que en ambos casos la incidencia de casos de disfunciones sexuales son similares.

En la Tabla 02, los factores presentes en la disfunción sexual fueron la depresión en un 18,3\%, El 8,3\% presentó depresión y disfunción sexual, con una OR $=1.43$ indicando que la depresión es un factor de riesgo de la disfunción sexual.

El 41,7\% presentó ansiedad; de estos el 16,7\% presentó Ansiedad y disfunción sexual; con una $\mathrm{OR}=$ 1,128 , significa que la ansiedad es un factor de riesgo de la disfunción sexual.

E1 5\% que utilizó antidepresivos presentaron disfunción sexual; Con una $\mathrm{OR}=0,96$ el mismo que significa que el uso de antidepresivos es un factor protector de la disfunción sexual.

Debemos resaltar que la depresión se asocia estrechamente con la disfunción sexual femenina. Además, la disfunción sexual es un efecto secundario frecuente de los antidepresivos, particularmente de ciertos inhibidores de la recaptación de serotonina. Una relación emocional satisfactoria con la pareja y el bienestar emocional global son buenos predictores de la ausencia de disfunción sexual.

Según Cáceres, (2012), En su estudio titulado "Función sexual y Factores de riesgo de la disfunción sexual de parejas sexuales que realizan un turismo sexual, Sus resultados evidencian que: Existe un 30\% de la población con disfunciones sexuales, y el 70\% tiene un funcionamiento sexual normal, Entre los factores de riesgo de la disfunción sexual fueron: el uso de anticonceptivos orales y parenterales con una $\mathrm{OR}=1,86$; Las enfermedades crónicas con una $\mathrm{OR}=$ 2,26 .

Portilla, (2008). En su estudio Incidencia y Factores de riesgo de la disfunción sexual en mujeres trabajadoras sexuales del Condominio la Coruña, 2007, Sus resultados indican que: existe una tasa de incidencia de 104 x 1000 trabajadoras sexuales, Siendo los factores de riesgo: el uso de los anticonceptivos orales $\mathrm{OR}=1,098$, ansiedad OR $=$ 2,43 abuso sexual $\mathrm{OR}=3,22$, En conclusión existe un alto porcentaje de casos de disfunción sexual, los factores de riesgo podrían ser controlables.

Cajahuanca, (2005). En su estudio Factores de riesgo de la disfunción sexual, en las asistentes al servicio de ginecología del Hospital Departamental de Ica, Sus resultados demostraron que los factores de riesgo de la disfunción sexual son: el uso excesivo de 
anticonceptivos orales $\mathrm{OR}=1,32$, asociados a la ansiedad, depresión $\mathrm{OR}=2,16$ y 1,86 respectivamente; así mismo los mayores de 40 años $\mathrm{OR}=1,68$; el cansancio también es un factor de riesgo $\mathrm{OR}=6,58$.

Comparando los resultados del presente estudio con los resultados de Cáceres, Portilla y Cajahuanca son similares ya que en dichos estudios los factores de riesgo de la disfunción sexual son el uso de los anticonceptivos orales, los anticonceptivos parenterales, Así mismo las enfermedades crónicas, son consideradas como un factor de riesgo.

En la tabla 03 , el $56,7 \%$ presenta fatiga, el $25 \%$ presentó fatiga y disfunción sexual; Con una OR = 1,776 significa que la fatiga es un factor de riesgo de la disfunción sexual.

Existe un 48,3\% que presentó abuso sexual, así mismo el $20 \%$ presentó abuso sexual y disfunción sexual; Con una $\mathrm{OR}=1,283$ significa que el abuso sexual es un factor de riesgo de la disfunción sexual.

El 56,7\% tuvieron una experiencia sexual negativa, El $15 \%$ que tuvo una experiencia sexual negativa también presentó disfunción sexual; Con una $\mathrm{OR}=$ 0,366 el tener una experiencia sexual negativa pareciera que es un factor protector de la disfunción sexual.

E1 43,3\% presentó preocupación, El 26,6\% presentaron preocupación y disfunción sexual; Con una $\mathrm{OR}=6,171$ quiere decir que las mujeres que tuvieron una preocupación tienen 6,171 veces mayor riesgo de presentar disfunción sexual que aquellas que no lo presentaron.

El $45 \%$ presentaron falta de privacidad, El 11,7\% que presentaron falta de privacidad presentaron disfunción sexual; Con una $\mathrm{OR}=0,372$ cabe indicar que la falta de privacidad es un factor protector de la disfunción sexual.

Según Cáceres, (2012), En su estudio titulado "Función sexual y Factores de riesgo de la disfunción sexual de parejas sexuales que realizan un turismo sexual, Sus resultados evidencian que: Existe un $30 \%$ de la población con disfunciones sexuales, y el $70 \%$ tiene un funcionamiento sexual normal, Entre los factores de riesgo de la disfunción sexual fueron: el uso de anticonceptivos orales y parenterales con una $\mathrm{OR}=1,86$; Las enfermedades crónicas con una $\mathrm{OR}=2,26$, la depresión y la ansiedad con una $\mathrm{OR}=3,56$, así mismo la fatiga $\mathrm{OR}$ $=2,65$, el abuso sexual $\mathrm{OR}=2,07$ y la preocupación $\mathrm{OR}=4,27$.

Portilla, (2008), En su estudio Incidencia y Factores de riesgo de la disfunción sexual en mujeres trabajadoras sexuales del Condominio la Coruña, 2007, Sus resultados indican que: existe una tasa de incidencia de 104 x 1000 trabajadoras sexuales, Siendo los factores de riesgo: el uso de los anticonceptivos orales OR $=1,098$, ansiedad OR $=$ 2,43 abuso sexual $\mathrm{OR}=3,22$, En conclusión existe un alto porcentaje de casos de disfunción sexual, los factores de riesgo podrían ser controlables.

Cajahuanca, (2005). En su estudio Factores de riesgo de la disfunción sexual, en las asistentes al servicio de ginecología del Hospital Departamental de Ica, Sus resultados demostraron que los factores de riesgo de la disfunción sexual son: el uso excesivo de anticonceptivos orales $\mathrm{OR}=1,32$, asociados a la ansiedad, depresión $\mathrm{OR}=2,16$ y 1,86 respectivamente; así mismo los mayores de 40 años $\mathrm{OR}=1,68$; el cansancio también es un factor de riesgo $\mathrm{OR}=6,58$, En conclusión, el cansancio es un factor más predisponente que el uso de anticonceptivos orales, depresión, A mayor edad mayor probabilidad de presentar disfunción sexual.

Comparando los resultados del presente estudio con los resultados de Cáceres, Portilla y Cajahuanca ya que los factores de riesgo de la disfunción sexual son: las enfermedades crónicas, el uso de los anticonceptivos orales, uso de anticonceptivos parenterales, Así mismo la depresión, ansiedad, la fatiga, abuso sexual y preocupación.

La disfunción sexual es un síndrome en el que los procesos eróticos de la respuesta sexual, resultan no deseables para el individuo, en forma recurrente o persistente y cuya causa no se asocia con alguna enfermedad; de lo contrario, deberá especificarse como trastorno sexual, El trastorno sexual se diagnostica cuando existe alguna alteración clínicamente significativa, provocada sólo por los efectos de alguna enfermedad, sus manifestaciones clínicas son del mismo tipo e intensidad que las clasificadas como disfunción sexual, Por todo ello, es necesario hacer un diagnóstico diferencial en relación con una serie de cuadros clínicos que abarcan a las disfunciones sexuales y que son divididas en cuatro grupos: alteración del deseo sexual, alteración de la excitación sexual, alteración del orgasmo y alteración que causa dolor sexual.

Existe otro aspecto sobre los posibles factores de la disfunción sexual y han demostrado que las mujeres con problemas en su vida sexual, también tienen alteraciones psicológicas que requieren atención por su vulnerabilidad, Se han encontrado asociaciones con niveles altos de ansiedad, autoestima baja, comunicación marital deficiente, características predominantemente sumisas en función del género, nulas conductas asertivas, estilo de comunicación negativo, problemas conyugales, experiencias infantiles traumáticas y nula o deficiente 
información respecto de la sexualidad.

\section{CONCLUSIONES}

1. De los factores biológicos: las enfermedades crónicas, las Ooferectompias, los anticonceptivos orales y anticonceptivos parenterales no tienen ninguna relación con la disfunción sexual.

2. Los factores biológicos y los anticonceptivos orales son factores de riesgo de la disfunción sexual, mientras que la Ooferectomía y los anticonceptivos parenterales son considerados factores protectores de la disfunción sexual.

3. De los Factores Psicológicos: la Depresión, Ansiedad y el uso de antidepresivos no guardan ninguna relación con las disfunciones sexuales,

4. De los factores psicológicos: La Depresión y Ansiedad son factores de riesgo de la disfunción sexual, mientras que el uso de antidepresivos es un factor protector de la disfunción sexual.

5. De los factores interpersonales: la fatiga, abuso sexual y falta de privacidad no tienen ninguna relación con la disfunción sexual, Pero sí la experiencia sexual negativa y la preocupación.

6. Los factores interpersonales como la Fatiga, abuso sexual y la preocupación son factores de riesgo de la disfunción sexual; mientras que la experiencia sexual y la falta de privacidad son factores protectores de la disfunción sexual.

\section{RECOMENDACIONES}

El personal del Programa de Planificación Familiar debe sustentar intervenciones educativas con un carácter activo, permanente, equitativo e inclusivo, de calidad que potencie formas de vida y desarrollo de su sexualidad de manera plena, sana y responsable, atendiendo a sus necesidades y a las demandas del entorno social y ambiental, como vía de garantizar los niveles de independencia y autodeterminación que le permita desarrollar la capacidad de elegir libre y responsablemente los límites y derechos personales de sus sexualidad y el respeto a que los demás tengan igualdad de posibilidades y oportunidades sexuales.

El Hospital Regional Virgen de Fátima debe trabajar en estrecha relación con la Universidad Nacional Toribio Rodríguez de Mendoza de Amazonas, para fortalecer prevenir los casos de disfunción sexual de las usuarias del programa de planificación familiar y velar por los derechos de las usuarias, brindándoles una mejor calidad de vida.

Fomentar la organización y planificación del personal de salud, mediante las capacitaciones periódicas y tenerlos actualizados facilitando la atención y educación de la familia y comunidad y así ejecuten programas de educación de calidad que garanticen conocimientos sobre los temas de factores de riesgo de la disfunción sexual.

Incentivar a los estudiantes a realizar investigaciones similares, ya estos pueden ayudar a sensibilizar y crear diferentes estrategias, para prevenir los altos índices de disfunción sexual.

\section{REFERENCIAS BIBLIOGRÁFICAS}

Alayo, T, (2012), El comportamiento sexual y su vinculación con variables psicológicas y demográficas en estudiantes de quinto año de educación secundaria, Tesis presentada para optar el Título Professional de Psicólogo, UNMSM, Lima - Perú.

Alfonso, R, (2010), "Educación sexual comunitaria, apuntes para un marco referente I Parte", Edic 2da, Edit, Sexología y Sociedad, España.

Aramburú, C, (2010), Uno empieza a alucinar... Percepciones de los jóvenes sobre sexualidad y acceso a los servicios de salud: Lima, Cusco e Iquitos, Lima: Redes Jóvenes.

Arias, R, (2011), Función sexual en las mujeres de la clase media de la Ciudad de México, 2da, Edic, Edit, Redes Jóvenes, Teluca, México.

Arnao, J, \& Cabezudo, C, (2001), Relación de las drogas con las actitudes sexuales y vínculos de pareja, Un estudio cualitativo descriptivoanalítico en adolescentes mujeres de Lima, Perú, Revista de Investigación en Psicología.

Aurole, E, (2014), Introducción al estudio de la sexualidad humana, Antología de la sexualidad humana, Edit, CONAPO; México.

Aurole, M, (2014), Introducción al estudio de la sexualidad humana, Antología de la sexualidad humana, Edit, CONAPO; México.

Briones, G, (2012) "Métodos y técnicas de investigación para las ciencias sociales", Segunda Edición, Editorial Trillas - México, Cuarta reimpresión.

Caballero, G, (2009), Conocimientos y actitudes sexuales de los géneros en la salud 
reproductiva de los adultos jóvenes, Umbrales de investigación, México.

Cáceres, C, (2011), Salud sexual en una ciudad joven: un programa comunitario en salud sexual con y para los jóvenes, Lima, Perú.

Cedrés, S, (2015), Disfunciones sexuales femeninas, 2da Edic, Edit, Plenus, Centro médico sexológico, España.

Cortés, A,; Sordo, M,; (2009), Comportamiento sexual y enfermedades de transmisión sexual en adolescentes, Chile.

Díaz, G, (2011), El sexo, Manual práctico ilustrado para la relación sexual, España: Everest, Diccionario de cultura sexual, El ABC de la sexualidad: Manuela Ramos, Lima, Perú.

Gómez, I, (2010), Conductas sexuales en adolescentes, Sexología, V(1), 35-43, Revista sexual, mundos paradigmáticos.

Gonzales, E, (2015), Función sexual de las usuarias del Programa de Planificación Familiar, Hospital Regional Virgen de Fátima, Chachapoyas, 2015, Investigación realizada en la Universidad Nacional Toribio Rodríguez de Mendoza de Amazonas, Chachapoyas.

González, C, \& Rojas, R, (2012), Perfil del comportamiento sexual en adolescentes mexicanos de 12 a 19 años de edad, Resultados de la ENSA, Salud Pública de México.

Hernández, C, (2012), Características de comportamiento sexual en mujeres de la Ciudad de México, Salud Pública de México.

Hernández, S, (2010) "Metodología de la investigación cientifica", 4ta Edición, Editorial Impreandes Presencia S,A, Colombia.

Katchadourian, H, (2008), La terminología del género y del sexo, En H, A, Katchadourian (Comp,), La sexualidad humana: Un estudio comparativo de su evolución, México: FCE.

León, R, \& Cossio, A, (2010), Actitudes y comportamientos sexuales en un grupo de estudiantes universitarios de Lima Metropolitana, Revista de NeuroPsiquiatría, Perú.

Martín, L, \& Reyes, Z, (2013), Conducta sexual, en las mujeres, Un enfoque integral de promoción de salud, Revista Cubana de Salud Pública, Cuba.

Munarriz, R, (2012), Tratamiento de la disfunción sexual femenina, Revista Internacional de Androl.

Pino, E, \& Sepúlveda, P, (2012), Disfunción sexual femenina, Sexología y Sociedad, Madrid, España.

Sánchez, C, (2011), "Metodología y diseño en la investigación científica" Segunda Edición, Editorial Mantaro, Lima, Perú.

Supo, J, (2014), "Metodología de la Investigación científica", 4ta Edic, Editorial Universitaria, Yanahuara Arequipa, Perú. 